\title{
Experiencias de laboratorio sobre el calor con un artefacto hogareño en la formación del profesorado de Física
}

\author{
Ernesto Cyrulies iD \\ Universidad Nacional de General Sarmiento (UNGS). Buenos Aires. Argentina. \\ ecyrulie@campus.ungs.edu.ar
}

[Recibido: 12 mayo 2020. Revisado: 28 diciembre 2020. Aceptado: 1 febrero 2021]

\begin{abstract}
Resumen: Se presenta una secuencia de enseñanza con actividades experimentales desarrollada en una materia sobre actividades de laboratorio del Profesorado Universitario en Física en la Universidad Nacional de General Sarmiento (UNGS). En la misma se hizo uso de una plancha doméstica como recurso didáctico para contextualizar y problematizar contenidos en relación a la transferencia de calor. Se conformaron grupos de trabajo con consignas de diferente grado de apertura para su resolución.
\end{abstract}

Palabras clave: Actividades de laboratorio, formación docente en física, transferencia de calor

Laboratory experiences on heat with a home appliance in Physics teacher training

Abstract: In this work, a teaching sequence with experimental activities developed in a subject on laboratory activities of the University Professorship in Physics at the National University of General Sarmiento (UNGS) is presented. In that sequence, a domestic iron was used as a didactic resource to contextualize and problematize content in relation to heat transfer. Working groups were asked to work with tasks that involved different degrees of openness for their resolution.

Keywords: Laboratory activities, teacher training in Physics, heat transfer

Para citar este artículo: Cyrulies E. (2021) Experiencias de laboratorio sobre el calor con un artefacto hogareño en la formación del profesorado de Física. Revista Eureka sobre Enseñanza y Divulgación de las Ciencias 18(2),2202. doi: 10.25267/Rev_Eureka_ensen_divulg_cienc.2021.v18.i2.2202

\section{Introducción}

En la enseñanza de la física es recomendable diseñar entornos de trabajo para el aula y el laboratorio, en los cuales los alumnos aprendan en diferentes formatos. Uno posible es la enseñanza a través del planteamiento de situaciones problemáticas que involucren los contenidos prescriptos en el currículo; esto es fuertemente sugerido por diferentes autores (Martínez-Torregrosa 2005, Guisasola et al. 2011). Además es preferible que las actividades sean más investigadoras en un progreso pautado y gradual, factible en un marco escolar, y que fomenten la reflexión por parte del alumnado sobre las prácticas y sobre la naturaleza de la ciencia (Domènech Casal 2013). Planteadas así, estas actividades promueven la construcción de nuevos conocimientos conceptuales contextualizados en la resolución de un problema. Según Tenreiro Vieira y Marques Vieira (2006) en ese formato los alumnos son invitados a elaborar una estrategia para resolver problemas y dado que estas actividades de laboratorio no suelen acompañarse de un protocolo de resolución, permiten además desarrollar ciertas capacidades a través del aprendizaje de metodologías científicas, y junto a ello comprender los procesos y la naturaleza de la ciencia. No obstante, de acuerdo con Crujeiras y Jiménez Aleixandre (2015) y Reiser (2004) resulta necesario, y es lo que hemos considerado en el trabajo presentado, brindar un andamiaje regulado de forma tal que desarrolle una autonomía que no se logra de modo inmediato; de no hacerlo, los estudiantes podrían no ser capaces de resolver por sí mismos las consignas que presentan los problemas. 
El término "problema" que se ha tomado acá tiene un sentido amplio, y su resolución incorpora experimentos, observaciones, actividades de registro, etc. A través de estas acciones se espera que los estudiantes se involucren de manera más activa en el trabajo de la materia y puedan vivenciar los aspectos planteados anteriormente, motivados, en principio, por la relevancia que atribuyen a los contenidos (o a la metodología). Estas consideraciones enmarcan al trabajo en el concepto de ABP (aprendizaje basado en problemas) donde los estudiantes integran teoría y práctica propiciando un trabajo investigativo en situaciones problemáticas más o menos abiertas. Sumado a esto la conformación de grupos de trabajo, como aquí se ha propuesto, resulta ser una estrategia acorde a lo que plantean algunos autores (Perrenoud 2004, Tejada Fernández 2009, Gairín Sallan 2011), quienes sostienen que el entorno social promueve la construcción de competencias específicas. Y, además, el rol docente sigue siendo central en el desarrollo de las mismas. Asumimos que el trabajo colaborativo promueve una mayor diversidad de aprendizajes; según Baker (2009) sucede porque ocurren procesos interaccionales que no se dan en situaciones de estudio individual.

En este caso el profesor cumple un rol diferente (aunque el aprendizaje se base en las actividades de los alumnos no significa que el docente sea pasivo), pues a menudo ejerce de facilitador o tutor, lo que hace que su labor no esté centrada en la explicación. El ABP tiene un enfoque centrado en el alumno, pero requiere una importante participación del docente (Rodríguez-Arteche, Martínez-Aznar 2016). Esta labor demanda una guía durante el desarrollo de las actividades promoviendo el rol activo que deben asumir los estudiantes en la resolución de los problemas. Esto se puede entender mejor en términos de comunicación metacognitiva. $\mathrm{El}$ docente plantea preguntas a los estudiantes que les ayuden a cuestionarse y encontrar por ellos mismos el mejor camino para el entendimiento y manejo del problema. Según algunos autores (Pozo y Carretero 1987), el hecho de aprender las cosas haciéndolas y encontrando sus propias soluciones hace más probable que las recuerden.

Una consideración especial merece tener, dentro de la enseñanza de la física en el laboratorio, el uso de los dispositivos tecnológicos conocidos por los alumnos como recursos didácticos particulares. Si éstos son objetos cotidianos pueden ser de gran utilidad potencial en términos de la didáctica disciplinar (Cyrulies 2017). Su inclusión en la enseñanza dota de sentido al estudio formal de los contenidos y brinda más posibilidades de aprendizaje, debido a que el alumnado puede relacionar estos contenidos con su vida cotidiana. Contemplamos aquí una mirada que vincule aspectos técnicos de diseño de los dispositivos con los propósitos formativos de los estudiantes. Una visión más conceptual de esta clase de dispositivos ya ha sido abordada por Hacking (1983), quien los denomina "theory made objects" (objetos hechos de teoría). Los mismos sólo pueden ser utilizados y comprendidos habiendo conceptualizado previamente fenómenos conocidos y definidos por la ciencia. Séré (2002) retoma esta idea planteando que en las manipulaciones que los estudiantes realizan en el laboratorio se ven forzados a utilizar conceptos estudiados, de este modo se tiene la "teoría al servicio de la práctica". Este tipo de objetos presentan mayores significados donde los conocimientos teóricos propios del ámbito científico regulan el marco metodológico de su uso.

Por otra parte, es conocido que el contexto de trabajo actual de un docente de secundaria plantea grandes retos. Los adolescentes de hoy en día tienen mucho acceso a otras formas de alcanzar información y conocimiento, por lo cual es parte del desafío diario que el docente plantee situaciones que resulten de interés para ellos. La física cobraría más sentido para los alumnos si les permitiera encontrar explicaciones sobre el mundo cotidiano; los artefactos que se utilizan en el hogar pueden ser un buen medio para esta posibilidad. Si este sentido se enriquece, da más posibilidades de lograr interés y alcanzar un aprendizaje más significativo en los alumnos. Esta enseñanza que atiende a lo cotidiano también es considerada en el "enfoque basado en el contexto" y CTS (ciencia, técnica y sociedad), donde las aplicaciones de la ciencia 
son un medio para desarrollar la comprensión científica. Diversos estudios concluyen en que dicho enfoque mejora las actitudes hacia el aprendizaje de la ciencia, particularmente en la escuela secundaria (Bennett, Lubben y Hogarth 2007). Pero una enseñanza basada en este tipo de prácticas requiere de competencias específicas, sobre todo del tipo experimental.

\section{Una materia que construye estas competencias}

La Universidad Nacional de General Sarmiento (UNGS) cuenta con la carrera Profesorado Universitario en Educación Superior en Física. Durante su desarrollo los estudiantes participan de distintas propuestas de laboratorio en las materias disciplinares con las que adquieren diversas competencias. Dado que el destino laboral de sus egresados incluye la enseñanza en el nivel medio, se incorporó al plan de estudios un espacio que atiende a las demandas que surgen de dicho nivel sobre la enseñanza de la física, promoviendo el desarrollo de competencias específicas en la formación docente. Se trata de la materia que se denomina Laboratorio para la Escuela Secundaria, la que se transita habiendo cursado algo más de la mitad de la carrera. Entre los propósitos de la misma se tiene promover un andamiaje conceptual que permita a los estudiantes tomar decisiones en función de las opciones metodológicas más adecuadas respecto a la enseñanza de contenidos que recurran a prácticas experimentales. En relación a ello, se espera el desarrollo de habilidades que les permita la construcción de diversos dispositivos con materiales comunes como el uso de artefactos cotidianos para la enseñanza evitando limitar las actividades de laboratorio al uso de equipos comerciales. Las actuales orientaciones didácticas de la escuela secundaria exigen prácticas que no demanden necesariamente material específico de laboratorio y no por ello carecerían de significatividad.

Entre otras propuestas de la materia se tiene la enseñanza de la física a través del planteamiento de situaciones problemáticas y la elaboración de investigaciones escolares que involucren los contenidos prescriptos en el currículo. Entendemos que una adecuada problematización de los mismos permite el desarrollo de habilidades más generales sobre prácticas relacionadas con actividades experimentales en el aula y en el laboratorio de física. Si bien la materia está atravesada por un marcado trabajo con conceptos de la disciplina se encuadra en una mirada didáctica que incluye la discusión y análisis crítico de propuestas de actividades contextualizadas, mediante el intercambio y aportes entre el grupo de estudiantes y los docentes, así como la implementación y registro de actividades de laboratorio elaboradas por los estudiantes.

\section{Descripción de la actividad}

La posible innovación que aquí se presenta consiste en tomar un artefacto hogareño y de uso cotidiano para contextualizar contenidos de la física e incluyendo -algunas de las- consignas en un formato investigativo. Se eligió una plancha doméstica por ser un elemento muy conocido y prácticamente presente en todos los hogares. Además, tiene la ventaja de ser sumamente simple en su funcionamiento lo que lo hace cognoscible sin necesidad de mayores conocimientos técnicos. En la secuencia se propone trabajar competencias en el laboratorio relacionadas a los temas abordados, pero que pueden ser pertinentes para guiar el trabajo de otros temas de la física.

La unidad didáctica trabajada (dos clases de cuatro horas) consiste en una secuencia con una marcada actividad experimental, procesamiento y análisis de datos. Se conformaron dos grupos de trabajo con los 8 estudiantes que completaban el curso (4 integrantes en cada uno y de ambos sexos). Se establecieron trabajos comunes a todos y otros diferenciados por grupo, de modo sincrónico, con una puesta final en común donde ambos grupos intercambiaron resultados y aprendizajes. La planificación contempló actividades con situaciones problemáticas planteadas a través de algunas consignas escritas. Algunas apelan a situaciones 
imaginarias para ejemplificar escenarios de investigación escolar y otras se plantean en un formato más dirigido o cerrado. Para ciertas consignas optamos por incorporar lo que Pifarré y Sanuy (2001) denominan "hoja para pensar el problema". Consiste en una presentación escrita o gráfica que permite orientar hacia una estrategia de resolución, pero sin consistir en una guía cerrada para resolverla. Además, en el presente trabajo se transcriben algunas de las preguntas orales posteriores que permitieron guiar la tarea.

Nota: La actividad no se propuso para ser transferida directamente a la escuela secundaria por desestimarse las mediciones con $220 \mathrm{~V}$ con los alumnos. Se planteó con estudiantes universitarios contando con las condiciones adecuadas de seguridad. Se asume que las instituciones educativas cuentan con dichas condiciones, sin embargo, las prácticas se realizaron con un dispositivo de protección extra para ser usado en la mesa que incluye disyuntor diferencial y llave térmica de $15 \mathrm{~A}$.

\section{Contenidos de la secuencia de actividades}

Los contenidos trabajados pueden dividirse en dos campos, los disciplinares propios de la física, estudiados en espacios anteriores del grado universitario y los correspondientes a la didáctica específica.

De la física (Termodinámica):

Transmisión del calor, energía interna, temperatura, ecuación de Newton del enfriamiento. Conservación y degradación de la energía. Energía eléctrica, consumo eléctrico. Intercambio de energía por radiación, emisividad.

\section{De la didáctica específica:}

Aprendizaje basado en problemas. Importancia pedagógico-didáctica de las actividades experimentales en la resolución de problemas y el modelo investigativo. Uso de las TIC en la enseñanza de la física.

\section{Implementación de la actividad}

\section{$1^{a}$ actividad en común: reconociendo la física en la tecnología}

Iniciando la primera clase se dispusieron un par de planchas en el laboratorio para su prueba de funcionamiento. Luego, se pidió a los estudiantes analizar el modo de trabajo de una plancha típica a partir de un dibujo incluido en fotocopias acompañado por unas preguntas para orientar el análisis (figura 1). En esta etapa de trabajo se propusieron una serie de cuestiones cuyo objetivo fue poner de manifiesto la relación de diferentes partes del dispositivo estudiado con ciertos contenidos de física.

\section{DETALLES INTERNOS DE UNA PLANCHA}

1. ¿Por qué calienta la plancha? ¿qué efecto eléctrico conocido se está aprovechando? ¿Podrian identificar en el dibujo el elemento responsable de eso?

2. Con la perilla 6 se selecciona el tipo de prenda, preestableciendo diferentes valores de temperatura. ¿Cómo logrará modificarla? ¿Observaron que tiene una luz piloto que enciende y apaga durante el funcionamiento?

3. Discutan en grupo la siguiente afirmación:

"La energía que ingresa a la plancha por suministro eléctrico es igual a la energía perdida por transmisión del calor."

4. Planteen sus hipótesis sobre los mecanismos de transmisión de calor involucrados. Piensen en estos dos casos: cuando está planchando una prenda y cuando se encuentra reposando en posición vertical. ¿Se transmitirá igual en ambos casos? ¿Qué mecanismo prevalecerá? ¿Estarán presentes los tres? (conducción, radiación y convección).

Figura 1. Fotocopia entregada a todos los estudiantes para la primera actividad. Fuente de la imagen: https://patentados.com/2005/plancha-a-vapor 
En la pregunta 2, se detectó desconocimiento sobre la existencia de un bimetálico, aun cuando resulta bastante evidente que alcanzada cierta temperatura algún mecanismo interrumpe la corriente eléctrica. Aquí el docente debió explicar que se hace uso del diferente coeficiente de dilatación de dos metales distintos, lo que curva el elemento que hace de interruptor. En esta etapa resultó interesante descubrir que no resulta obvio cómo opera el mecanismo de regulación de la temperatura, aun para estudiantes avanzados de la carrera. Varios de los asistentes suponían que se trataba de un sistema de resistencia variable, a modo de reóstato (se explicó lo inviable que resulta para el caso). Se mostró un dispositivo llevado para la ocasión (figura 2).

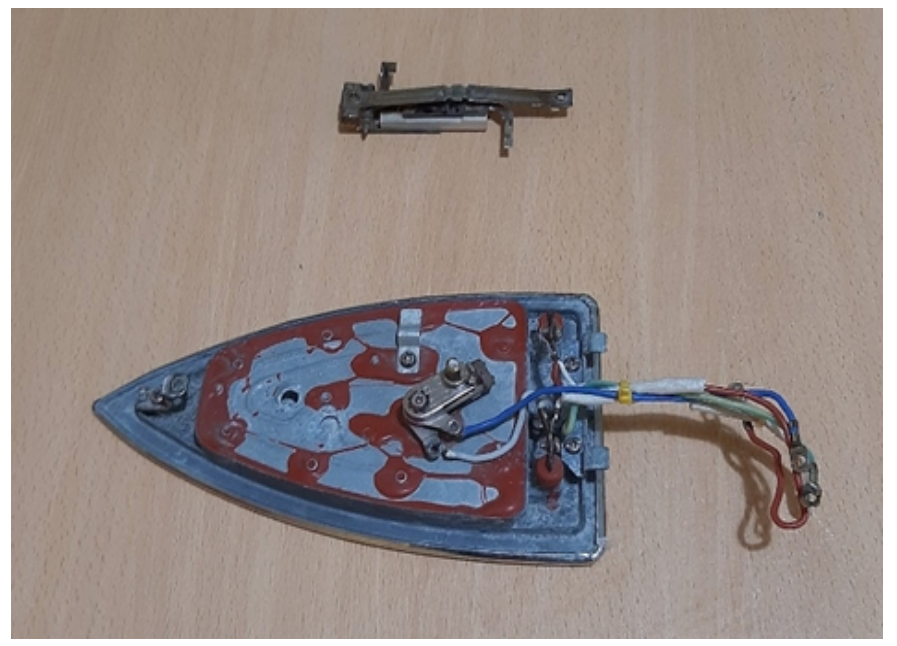

Figura 2. Chasis de plancha en desuso utilizado para observar el interruptor automático y analizar su funcionamiento. A su lado, otro modelo de interruptor utilizado por otras marcas comerciales. Puede descubrirse observando el chasis que la resistencia responsable del calentamiento se encuentra integrada y no resulta visible.

El punto 3 permitió distinguir un proceso de intercambio de energía considerando un instante de otro donde se toma en cuenta el mediano plazo teniendo en cuenta el funcionamiento intermitente de esta clase de aparatos. La afirmación planteada en la consigna no vale por igual para uno u otro caso. Esta consideración resultó relevante para una actividad posterior detallada más adelante.

El punto 4 dio lugar a un primer análisis sobre los mecanismos de transmisión de calor, pero se profundizó más tarde en actividades que se describen más abajo.

\section{$2^{a}$ actividad en común: la variación de la temperatura}

Se conectó una plancha y se registraron los valores de temperatura a lo largo de un período de tiempo tal que permitió construir una curva de calentamiento y enfriamiento (figura 3). Para esto se utilizó una placa Arduino Uno con un sensor DS18B20. Se realizó un análisis cualitativo de dicha curva.

El ensayo permitió observar que la temperatura oscila de modo periódico, con una variación aproximada de unos $8{ }^{\circ} \mathrm{C}$ según registros de los estudiantes. Con algunas pruebas se comprobó también que el período depende de la posición de la perilla de regulación. La hipótesis acordada por los estudiantes fue que con temperaturas más altas se hace mayor la diferencia con la del ambiente aumentando así la transferencia de calor en el período de enfriamiento. Esto acorta el período entre un encendido y el siguiente. El gráfico obtenido muestra además algo previsible en cada ciclo, que es que el calentamiento es más rápido que el enfriamiento. El programa fue elaborado por la cátedra en Open hardware. 


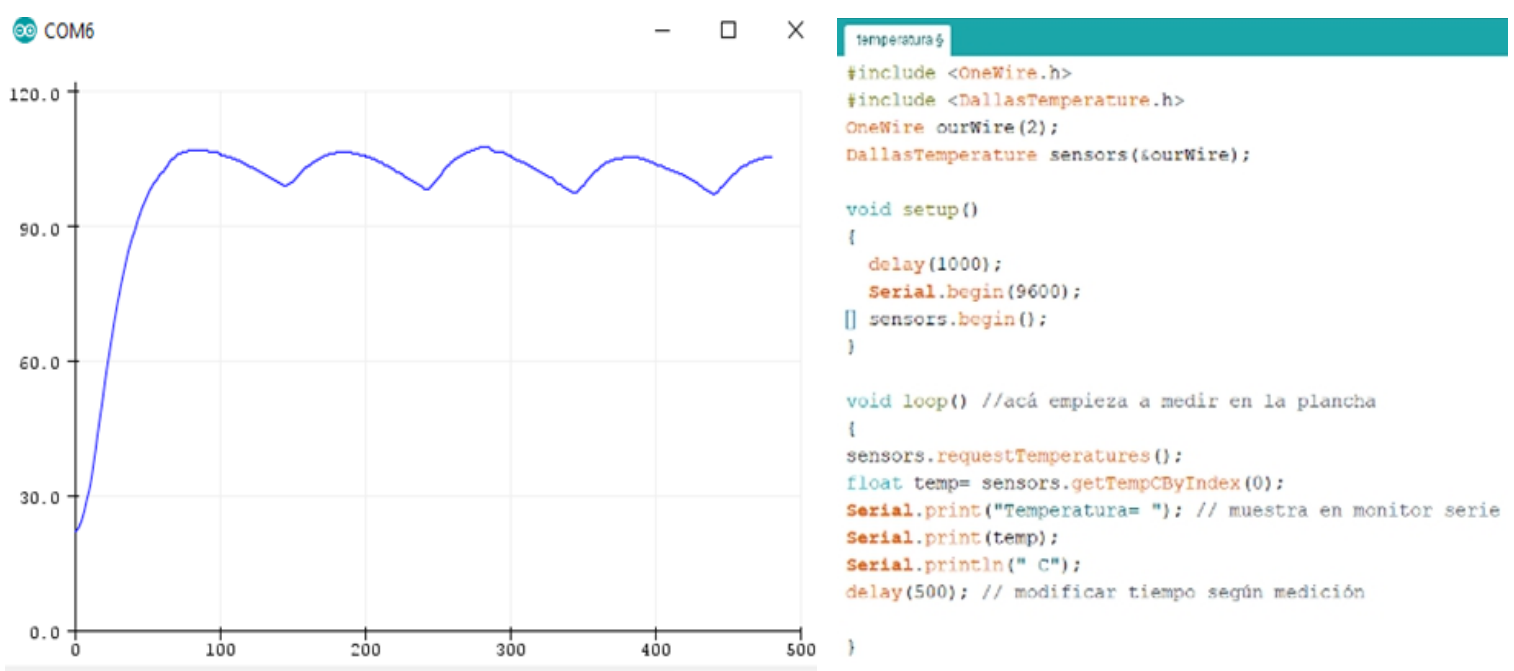

Figura 3. Izquierda: Curvas de temperatura de calentamiento y enfriamiento durante algunos ciclos de funcionamiento que inician en la temperatura ambiente con la conexión eléctrica (temperatura en grados centígrados, tiempo en segundos) Derecha: Programa para la medición de temperatura en el entorno de Arduino.

\section{$3^{a}$ actividad en común: la relación con la radiación}

Se reparte el siguiente material (figura 4). Con el mismo se espera recuperar saberes de los estudiantes para que puedan relacionarlos con actividades posteriores.

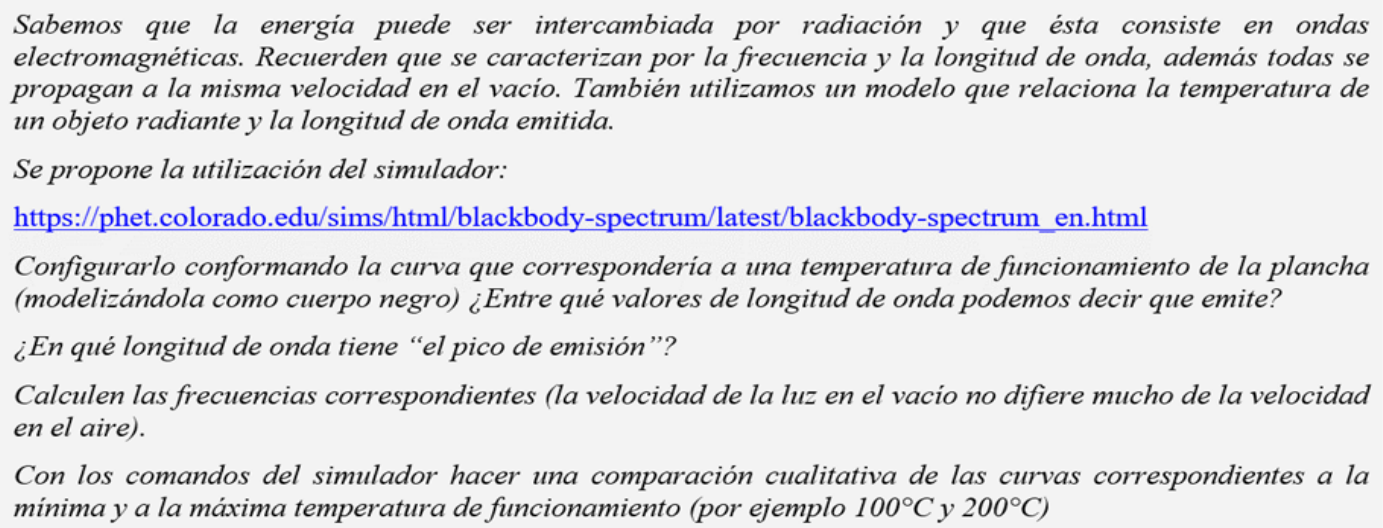
electromagnéticas. Recuerden que se caracterizan por la frecuencia y la longitud de onda, además todas se propagan a la misma velocidad en el vacio. También utilizamos un modelo que relaciona la temperatura de un objeto radiante y la longitud de onda emitida.

Se propone la utilización del simulador:

https://phet.colorado.edu/sims/html/blackbody-spectrum/latest/blackbody-spectrum_en.html

Configurarlo conformando la curva que corresponderia a una temperatura de funcionamiento de la plancha (modelizándola como cuerpo negro) ¿Entre qué valores de longitud de onda podemos decir que emite?

¿En qué longitud de onda tiene "el pico de emisión"?

Calculen las frecuencias correspondientes (la velocidad de la luz en el vacio no difiere mucho de la velocidad en el aire).

Con los comandos del simulador hacer una comparación cualitativa de las curvas correspondientes a la minima y a la máxima temperatura de funcionamiento (por ejemplo $100^{\circ} \mathrm{C}$ y $200^{\circ} \mathrm{C}$ )

Figura 4. Recuperación de conceptos y propuesta de modelización con simulador

En la figura 5 puede verse una figura que se obtuvo con el simulador configurado a una temperatura dentro del rango establecido como parte de lo propuesto en la actividad.

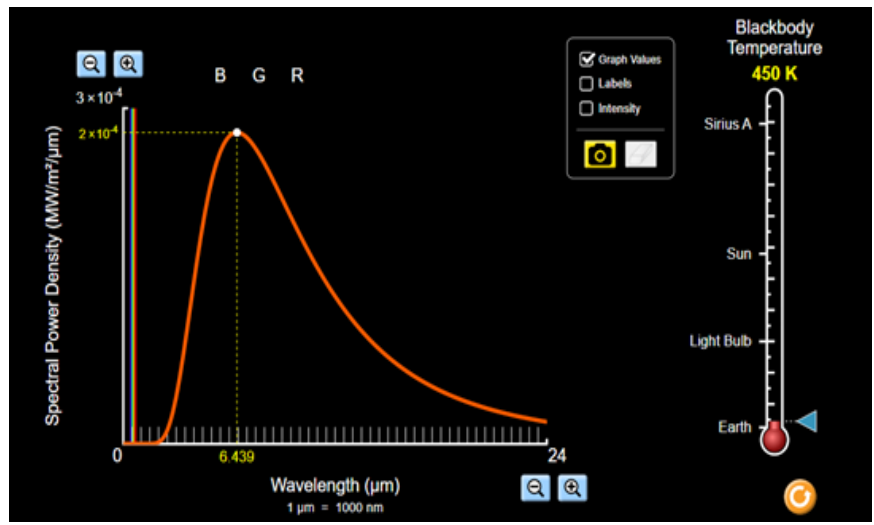

Figura 5. Curva de emisión de un cuerpo negro obtenida con el simulador PHET para la temperatura de la plancha. 


\section{$4^{a}$ actividad en común: la relación con la convección}

Después de la modelización de la radiación se presenta una consigna más guiada (figura 6), relacionada con la anterior, para conseguir valores de temperatura y es introductoria a otra actividad de la secuencia.

- Medir la temperatura en un punto cercano a la base de la plancha en posición horizontal, con ésta apuntando hacia arriba y luego hacia abajo.

- Dejar que la plancha alcance su temperatura de trabajo.

- Anotar los valores registrados en el termómetro para ambas configuraciones.

- Importante: El termómetro debe permanecer a la misma distancia de la base $(\sim 1,5 \mathrm{~cm})$

Figura 6. Comprobación de los efectos de la convección

Pregunta a todo el grupo:

¿La cantidad de energía transferida por radiación cambiará rotando la plancha?

Los intercambios con los estudiantes mostraron que sabían que esto no ocurre dado que se trata de ondas electromagnéticas, las que no se ven afectadas por la gravedad (a diferencia de los movimientos convectivos); no obstante, reconocieron que la actividad resulta una oportunidad para discutir que, a dichas ondas, al no tratarse de algo sustancial, no se les atribuye fuerza gravitatoria.

La experiencia mostró que el aire calentado en las cercanías de la superficie tiene un comportamiento claramente distinto en ambos casos y, la gravedad acá sí tiene influencia. Permitió concluir a los estudiantes que la convección es la causa de la diferencia de temperatura que registraron en ambas mediciones. Se obtuvieron resultados convincentes con la distancia sugerida en la consigna. Para un caso se registró $43^{\circ} \mathrm{C}$ y para el otro $27^{\circ} \mathrm{C}$ con la plancha en régimen de funcionamiento. En la figura 7 se adjuntan fotos de ambas posiciones.
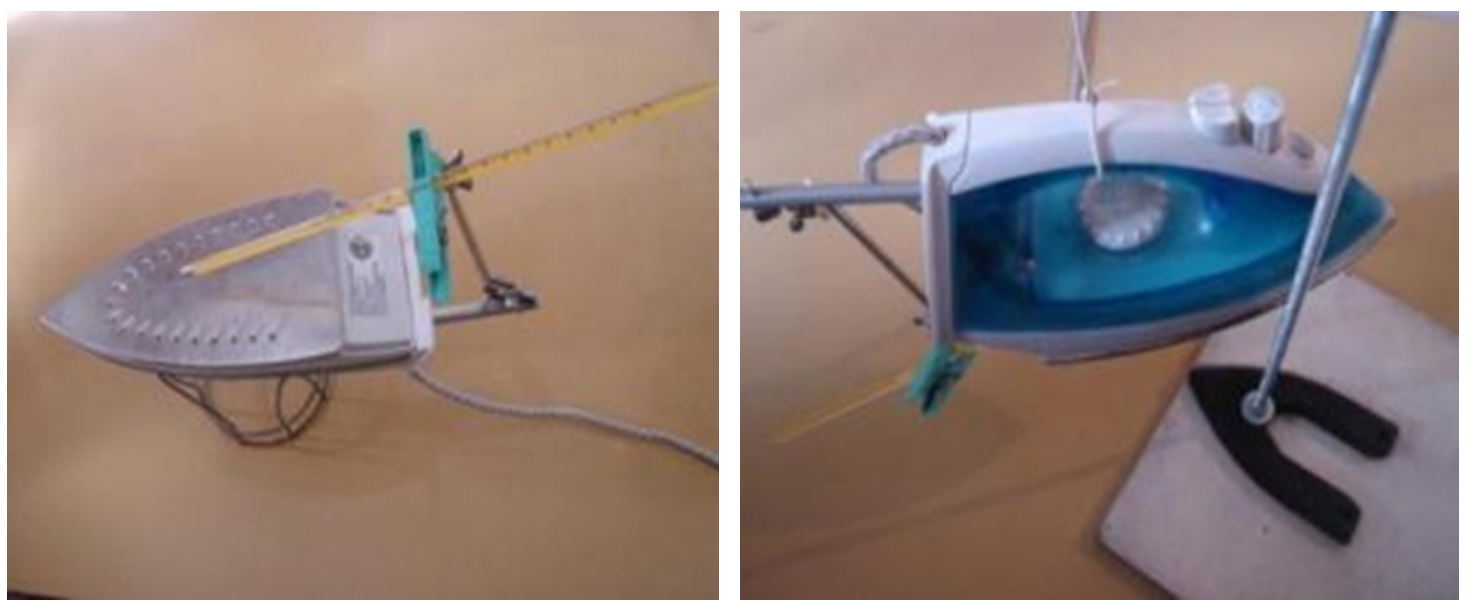

Figura 7. Posiciones de la plancha para mostrar la acción de la convección. En ambas situaciones se conserva la posición relativa del termómetro respecto de la base.

Finalmente se realizó en clase una demostración interesante que no fue fotografiada. Consistió en interponer la plancha caliente entre el cañón de proyección utilizado y la pantalla. Esto permitió observar en esta última con mucha claridad la turbulencia en el aire que caracteriza a la convección (debido a su cambio en el índice de refracción por la temperatura).

\section{Actividades diferenciadas por grupo}

Como se ha anticipado, se propuso dividir a los estudiantes en dos grupos con dos consignas de trabajo distintas en torno a una línea común en un formato investigativo. A cada grupo (en 
adelante $\mathrm{A}$ y B) se le entregó una problemática específica que requirió diseñar estrategias experimentales. Si bien éstas fueron libres, se concibieron a partir de una problemática estratégicamente elegida. Por ello, dadas las respuestas cuantitativas demandadas, las estrategias no deberían ser demasiado diferentes a las que aquí se presentan. Además, como cabe esperar en toda intervención didáctica, se sostuvo cierta orientación docente para desarrollar estas acciones. Las actividades requirieron mediciones eléctricas con instrumentos lo cual exigió un debido monitoreo y control.

En la figura 8 se muestran las situaciones planteadas que se entregan a todos los estudiantes y que presentan un contexto imaginario común. Se propone realizar una actividad experimental en el laboratorio que permita responder a las preguntas
dadas a cada grupo. Para iniciar la actividad, discutan en el grupo las mediciones y cálculos que harian
para responder las preguntas.
Materiales con los que contamos:
Plancha hogareña
Algunas prendas
Termómetro digital con termocupla
Amperímetro- voltímetro (multímetros)
Teléfono celular con cronómetro y cámara fotográfica
Cables para conexiones
Netbook
Factura de consumo eléctrico
Protecciones eléctricas (disyuntor diferencial y llave térmica 15 A)
SITUACIÓN PROBLEMÁTICA
Una empresa nacional comenzará la fabricación de muevas planchas y en la misma desean saber si pueden
mejorar las prestaciones de las existentes en el mercado. En el departamento de diseño se enteran de que en
la presente materia del profesorado de fisica asisten estudiantes capaces de resolverles los siguientes
interrogantes:

Figura 8. Propuesta de trabajo integradora

Para el grupo A las directrices comunes se complementan con el siguiente material:

\section{SITUACIÓN PROBLEMÁTICA PARA TRABAJAR EN EL GRUPO A}

A1- ¿Cuánto dinero cuesta un planchado normal de prendas durante una hora? Tengan en consideración que la plancha no toma energía de la línea de modo permanente.

A2- Al desenchufarla, ¿de qué modo se enfria y cuánto hay que esperar para guardarla? (Una vez consensuada la temperatura máxima para hacerlo) Se requiere modelizarlo matemáticamente.

Discutan en grupo la forma de registrar la disminución de temperatura.

Figura 9. Propuesta diferenciada (A)

Para el grupo B se entrega la siguiente situación:

\section{SITUACIÓN PROBLEMÁTICA PARA TRABAJAR EN GRUPO B}

B1- Cuando planchamos, ¿qué porcentaje de la energía eléctrica que consume la plancha es disipada a través de la convección mientras la depositamos parada sobre la tabla?

B2-Si las planchas se fabrican de menor peso bajan los costos de producción. ¿desde el punto de vista de su funcionamiento tendrá alguna ventaja para sus usuarios construirlas más livianas?

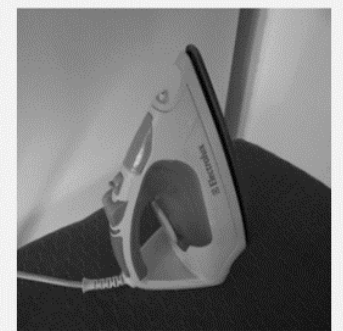

Figura 10. Propuesta diferenciada (B) 


\section{Sobre el trabajo del grupo A}

\section{Actividad A1: Planchado y mediciones en el laboratorio}

Con la propuesta se espera que los estudiantes concluyan que es necesario determinar la energía total consumida durante el planchado y que dicha energía deberá calcularse determinando la potencia media de la plancha. Para esto último, resulta necesario medir el tiempo empleado en la operación.

Si bien en la plancha puede estar indicada la potencia eléctrica, se consideró conveniente, junto a los estudiantes, determinarla con amperímetro y voltímetro. Por un lado, es posible que la plancha no tenga las características técnicas a la vista y, por otro lado, la potencia indicada puede diferir de la real (además ésta depende de la tensión eléctrica, la que suele tener variaciones). En este artefacto, para el circuito eléctrico puede considerarse un modelo puramente resistivo, lo cual podría ser una ventaja al simplificar el cálculo para corriente alterna.

En primer lugar, los estudiantes necesitaron conectar la plancha a los instrumentos eléctricos (figura 11). Se trabajó con el grupo sobre su uso cuidadoso y metódico tal que preserve dicho instrumental; se les solicitó que dibujaran las conexiones de manera simbólica. Esto puso en juego saberes sobre representaciones icónicas que son parte de las competencias comunicativas en el aula en su futura práctica docente.

Se propuso tomar tiempos con teléfono celular y registrarlos en una tabla. Se consensuó en una medición simultánea con varios teléfonos para luego tomar un promedio y se despreció el tiempo de respuesta en la manipulación de los mismos.

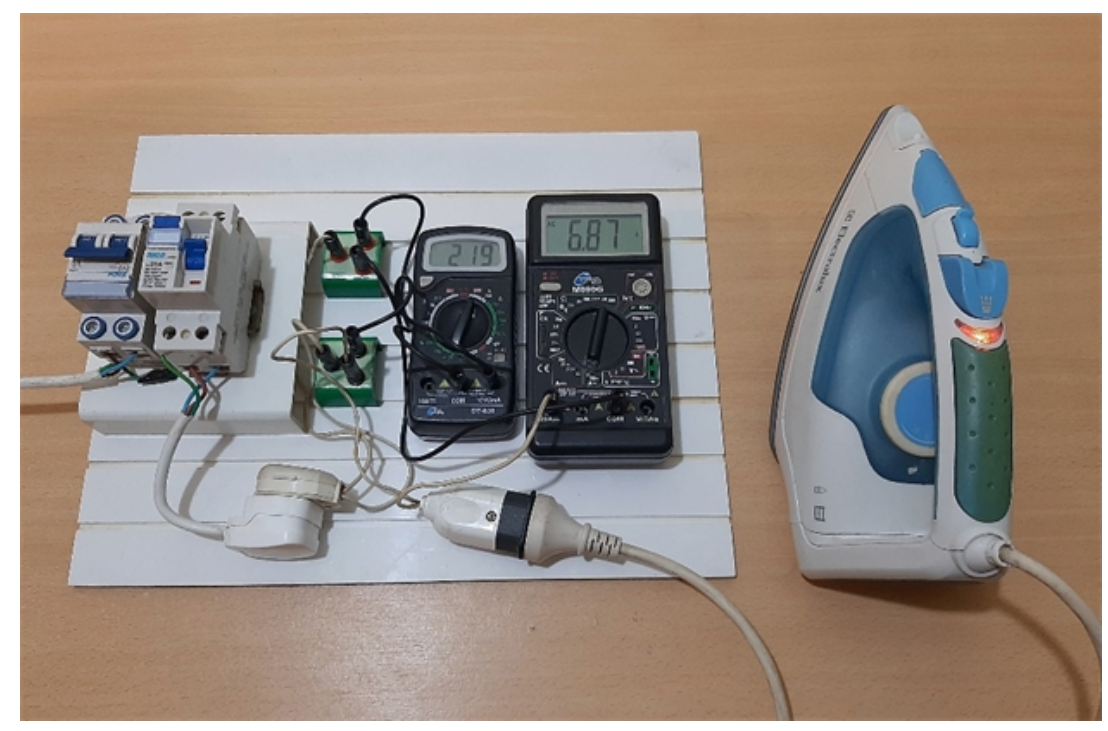

Figura 11. Disposición de los elementos de seguridad y de los multímetros utilizados en el laboratorio. Puede verse el registro en simultáneo de tensión y corriente.

Se tomó el tiempo de planchado de diferentes prendas llevadas para la ocasión atendiendo sólo a los intervalos en que el amperímetro acusó lectura (cuando no es así, el circuito eléctrico está interrumpido y no hay consumo). Simultáneamente se realizaron las mediciones eléctricas en la plancha para este problema y se acordó realizar la experiencia durante 15 minutos dada la intermitencia de su funcionamiento. Se comprobó que la potencia nominal indicada por los fabricantes es muy aproximada. 
Se calculó la energía a través de la expresión conocida por los estudiantes que se muestra en la ecuación 1.

$$
E=V \cdot I \cdot \Delta t \quad(1)
$$

donde $\Delta \mathrm{t}$ es la sumatoria de los tiempos parciales con piloto encendido, V es la tensión e I la corriente. De estas consideraciones surgió que para una plancha de $1500 \mathrm{~W}$ se tiene una potencia media de $525 \mathrm{~W}$ para las condiciones de ensayo en el laboratorio. Este último resultado surge como cociente entre la energía calculada y el tiempo total de uso (que incluye los tiempos de desconexión).

Por otra parte, las mediciones realizadas también dieron cuenta del poco cambio de la resistividad con la temperatura ( $2 \%$ en promedio) al medir la resistencia desde el enchufe de conexión (figura 12, izquierda). Esta medición permitió resolver el problema de un modo alternativo registrando el valor de la resistencia $R$ en caliente a través de la ecuación 2 .

$$
E=\left(\frac{V^{2}}{R}\right) \cdot \Delta t
$$

A continuación, conocido ya el valor de la energía necesaria para esta operación doméstica, los estudiantes calcularon el costo del kW.h a partir del consumo bimestral indicado en la factura disponible de la distribuidora eléctrica (ver figura 12, derecha). Como actividad extra se realizaron algunas consideraciones sobre el error en el resultado a partir de las incertezas en las mediciones.
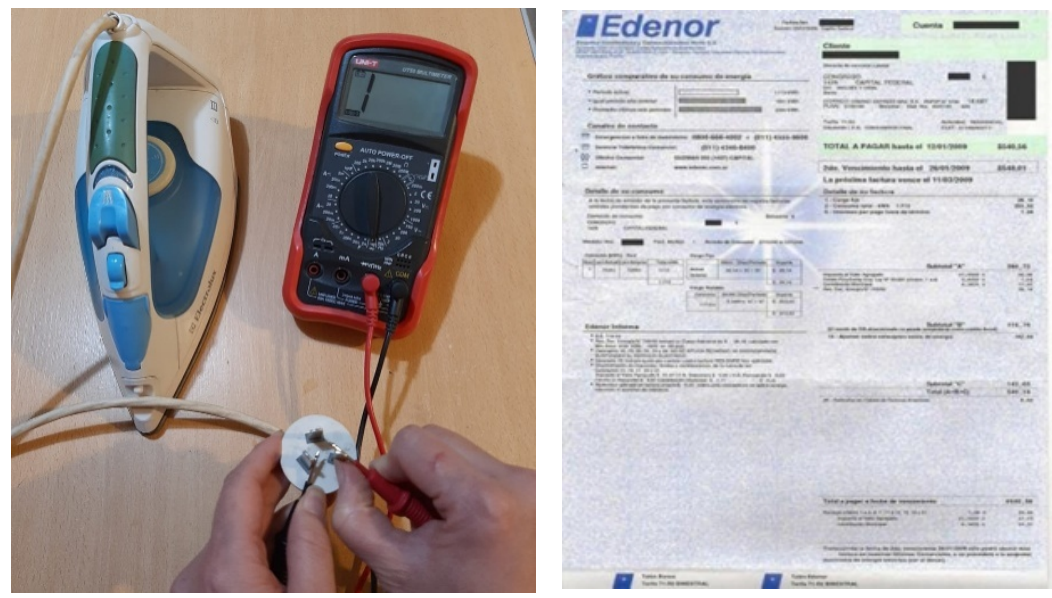

Figura 12. Izquierda: Medición de la resistencia a través de las clavijas del enchufe. Se incluyó en la medición la tercera pata para verificar el estado de la puesta a tierra del artefacto. Derecha: Factura de la empresa distribuidora de energía utilizada como fuente de datos.

Finalmente, se discutió la posibilidad de hacer estimaciones del costo total del planchado de toda la ropa de la familia, en el bimestre, etc. Fue oportuno recordar el uso coloquial de esta unidad física, dado que suele decirse "kilowatt" en vez de "kilowatt- hora" y la primera, en realidad, es unidad de potencia.

\section{Actividad A2: Construcción del modelo}

Sabemos de la teoría (ley de enfriamiento de Newton) que la temperatura de un cuerpo enfriándose en un medio puede modelizarse con una exponencial decreciente $\mathrm{T}(\mathrm{t})$, que es la solución de la ecuación diferencial correspondiente. Se propuso comprobar empíricamente con la plancha esta ley de enfriamiento. Para ello se sugirió medir la temperatura (con la plancha caliente pero desenchufada) a intervalos regulares de tiempo y graficar con algún software (Excel) que permita encontrar el modelo matemático. Se utilizó una termocupla para medir la temperatura a intervalos regulares de tiempo y, de forma adicional, el proceso se 
modelizó con el software libre Maxima, lo que permitió a los estudiantes encontrar la solución de la ecuación diferencial. Se entregó el material que se muestra a continuación (figuras 13 y 14).

\section{SUGERENCIAS PARA LA ACTIVIDAD A2 (GRUPO A)}

Calentar la plancha hasta su temperatura máxima y desenchufarla.

Medir temperaturas en intervalos regulares de tiempo durante un periodo que consideren adecuado. Registrar los datos.

Procesar los datos con planilla de cálculo y graficar buscando la función matemática que modelice adecuadamente la temperatura en función del tiempo.

Extrapolar la solución a tiempos mayores para evaluar si el modelo predice valores de temperatura que no se usaron para ajustar la función.

Figura 13. Sugerencias para la actividad A2

Se propone aquí considerar el caso del enfriamiento de un cuerpo por disipación hacia el medio que lo rodea. Recordemos el modelo que plantea la ley de Newton para el enfriamiento:

$$
\frac{d T}{d t}=-k\left(T-T_{m}\right)
$$

donde

T: temperatura

t: tiempo

$k$ : constante

La ecuación permite ver que la velocidad de enfriamiento de un cuerpo es proporcional a la diferencia de temperatura entre dicho cuerpo y la del medio que le rodea. En muchos casos esta última es la temperatura ambiente. Por otro lado, $k$ es la constante de proporcionalidad que depende de las condiciones y materiales involucrados.

La solución de la ecuación (encontrarla), gráficamente, se verá más o menos como el gráfico de la derecha.

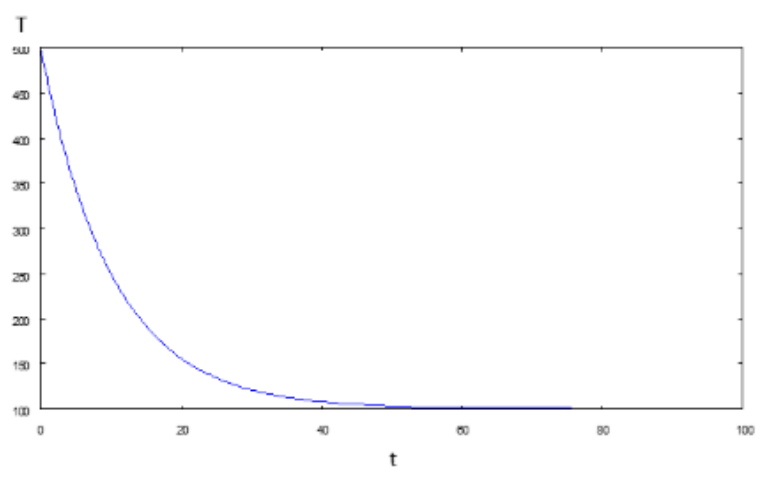

Figura 14. Apoyo teórico para la actividad A2

Una vez decididas las temperaturas con las que iniciar y finalizar la experiencia, así como los tiempos de medición, los estudiantes realizaron las mediciones de temperatura en intervalos regulares de tiempo utilizando termómetro y teléfono celular. Con las mediciones registradas construyeron una tabla en la planilla de cálculo con los datos de temperatura y tiempo y graficaron los puntos correspondientes ( $\mathrm{T}$ como función de $\mathrm{t}$ ). A continuación, encontraron una función matemática que se ajustó adecuadamente a dicha sucesión de puntos. Los estudiantes podrían haber utilizado cualquier función que aproximara a los datos, pero utilizaron una exponencial dado que conocían de la teoría que es la que modela un enfriamiento como el planteado en condiciones ideales. Para el caso real puede estar algo alejado de las condiciones ideales, por lo cual podría encontrarse una función polinómica que ajuste mejor. Sin embargo, no será la más conveniente si se desea despejar t para cierta $\mathrm{T}$, particularmente para un polinomio de grado mayor a dos, donde resultaría engorroso y no significaría mayor aporte al aprendizaje. Además, el modelo aceptado parte de que la tasa de 
enfriamiento es proporcional a la diferencia de temperatura lo que lleva a encontrar como solución una función exponencial decreciente.

Resultó importante poner en debate esta acción como una aproximación al trabajo de modelización en ciencia tomando como eje de trabajo la energía térmica. Se destacó la posibilidad de extrapolar los resultados de modo que pudieran determinar la temperatura probable para un tiempo posterior al utilizado para las mediciones (también se mencionó la interpolación entre dos valores medidos).

Se muestran a continuación, en la figura 15, los resultados obtenidos (cabe aclarar que en esta actividad se valoró la toma de datos en forma manual y no la precisión; la que hubiese sido mayor en el caso de usarse el dispositivo y el software de la segunda actividad en común).

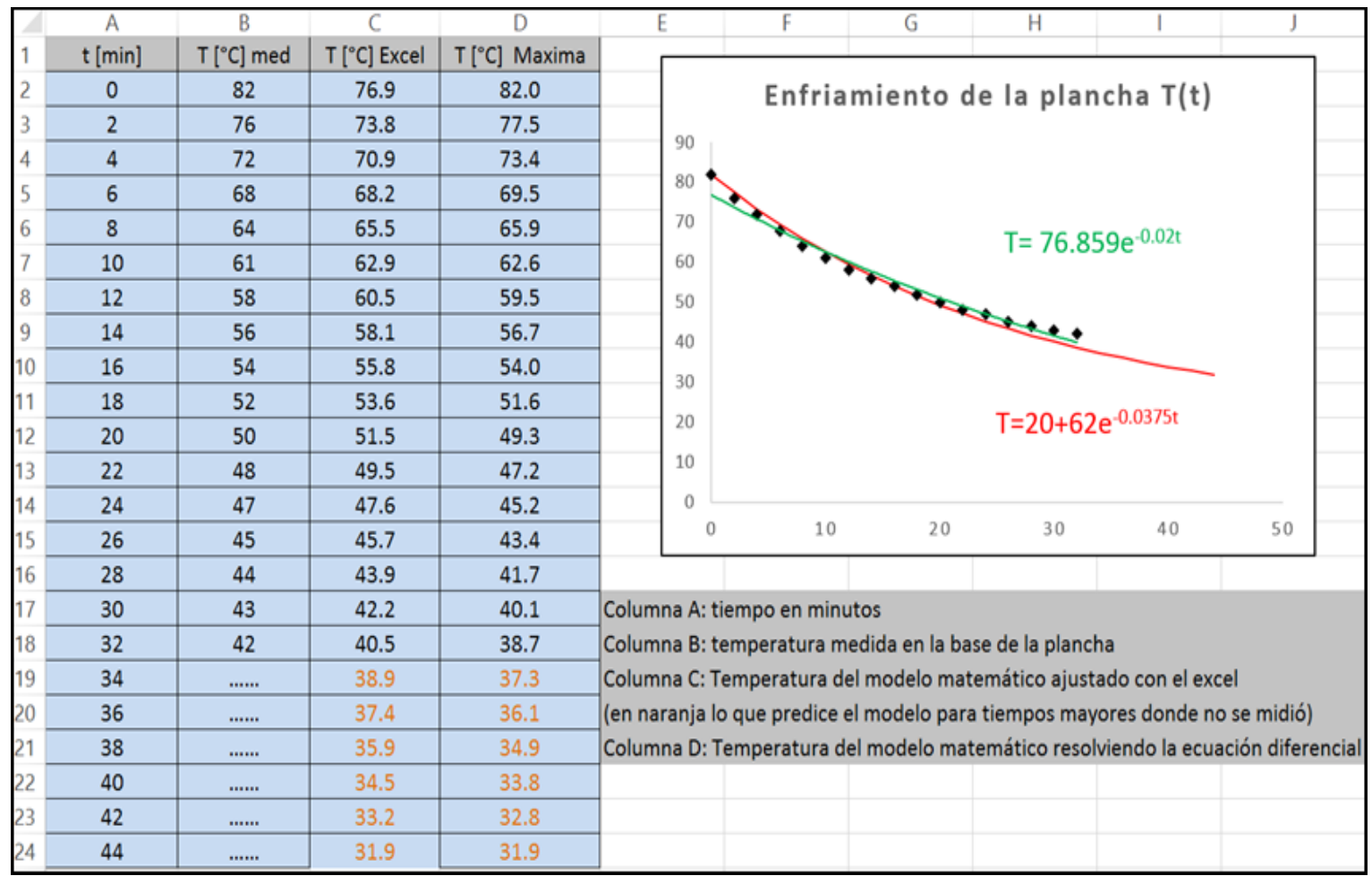

Figura 15. Planilla de cálculo que muestra el resultado a partir de los datos obtenidos. La cuarta columna, construida a partir del resultado de resolver la ecuación con Maxima revela cierta discrepancia con la función obtenida con el Excel. En naranja, datos obtenidos con la extrapolación correspondiente (tiempos no medidos). En el gráfico de la derecha pueden verse los modelos obtenidos a través de Excel (en verde) y de la resolución de la ecuación diferencial con Maxima (en rojo).

La extrapolación permitió predecir la temperatura de la plancha más allá del tiempo medido dando respuesta al problema. Se encontró la solución de la ecuación diferencial con Maxima a partir de las condiciones iniciales y un valor $\mathrm{T}(\mathrm{t})$ registrado, logrando un mejor ajuste para los primeros valores. Se asumió que la discrepancia con el modelo teórico obedece a errores experimentales y condiciones de contorno no contempladas; éstas fueron cuestiones de interés para discutir con los estudiantes. Si bien conocían la teoría de una asignatura anterior (Termodinámica y Fluidos) asumieron que allí se resaltó "el saber" en relación al contenido, lo cual se complementaría con los aspectos procedimentales de esta propuesta.

Se muestra a continuación, en la figura 16, las sentencias utilizadas en Maxima por los estudiantes y el gráfico obtenido. 

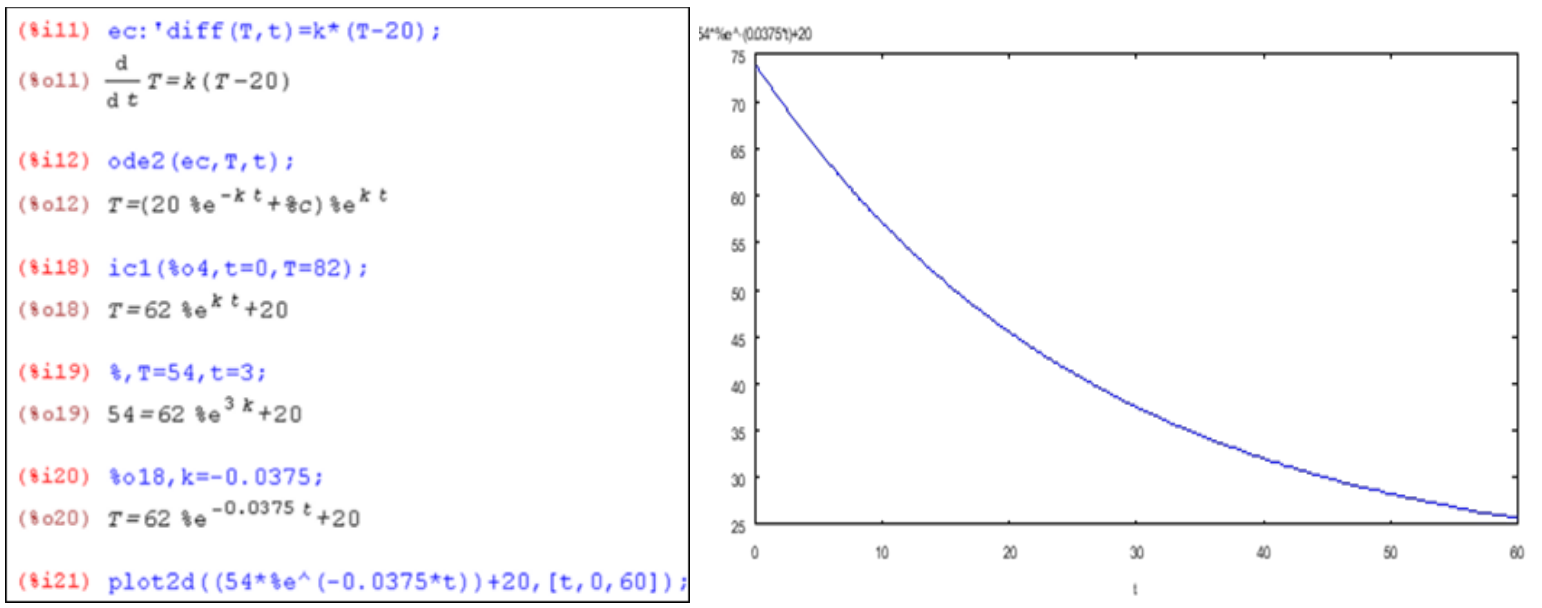

Figura 16. Izquierda: programa para modelizar la curva de enfriamiento. Derecha: curva obtenida con el programa.

\section{Sobre el trabajo del grupo B}

\section{Actividad B1: Determinación del valor de la convección}

Al inicio de esta actividad se entregó al grupo un breve escrito (figura 17) para poner en discusión una afirmación y brindar orientaciones.

\section{ORIENTACIONES PARA EL GRUPO B}

Discutir en el grupo la siguiente afirmación: La energía que ingresa a la plancha por suministro eléctrico es igual a la energía perdida por transmisión del calor.

Hipotetizar sobre los mecanismos de transmisión de calor presentes en la plancha. Pensar en dos casos: cuando se está planchando una prenda y cuando se deja reposando en posición vertical. ¿Se transmitirá igual en ambos casos? ¿Qué mecanismo prevalecerá? ¿Estarán presentes los tres?

Figura 17. Material entregado al grupo B

Se planteó aquí la determinación de una variable, cuyo cálculo no resulta abordable de forma directa sino a través de la medición de otras variables asociadas. Considerando que este proceso a menudo es propio del trabajo científico, aquí se convirtió en una estrategia didáctica para promover la imagen de ciencia que se pretende que los estudiantes adquieran.

Poder hacer mediciones para determinar la disipación de energía, aún en un artefacto sencillo como una plancha es dificultoso. De los tres mecanismos conocidos, claramente, el más complicado es la convección. Por otro lado, la conducción puede ser difícil de calcular debido a condiciones de frontera complejas en el contacto con la prenda. Entonces, a los efectos de que los estudiantes puedan cuantificar algún proceso de disipación, se sugirió considerar la radiación de la plancha sin contacto con la tela (de ahí surge la situación problemática presentada).

El método consistió en asumir que puede despreciarse la conducción del calor a través del aire. De este modo, se consideró que la energía eléctrica consumida por la plancha es disipada sólo a través de la convección y de la radiación. Dado que esta última es más sencilla de calcular, la convección se obtuvo restando a la energía eléctrica la disipada por radiación. La figura 18 muestra el esquema entregado para dicha deducción. 


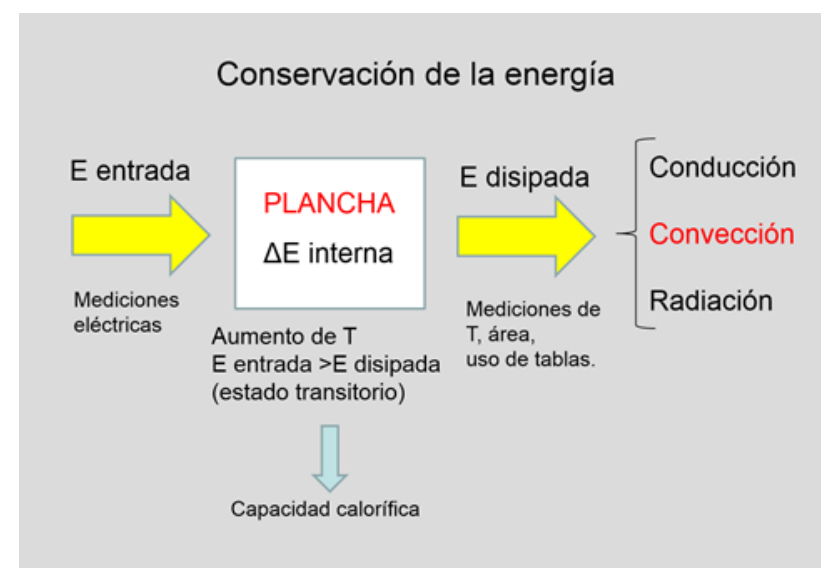

Figura 18. Esquema para "pensar el problema" compartido con el grupo B que representa los conceptos involucrados en la actividad B1.

Llegados a esta etapa los estudiantes del grupo B necesitaron calcular el valor de la radiación durante el funcionamiento a una temperatura elegida. Con la entrega de material extra (figura 19) se presentó teoría que permite modelizar la situación problemática.

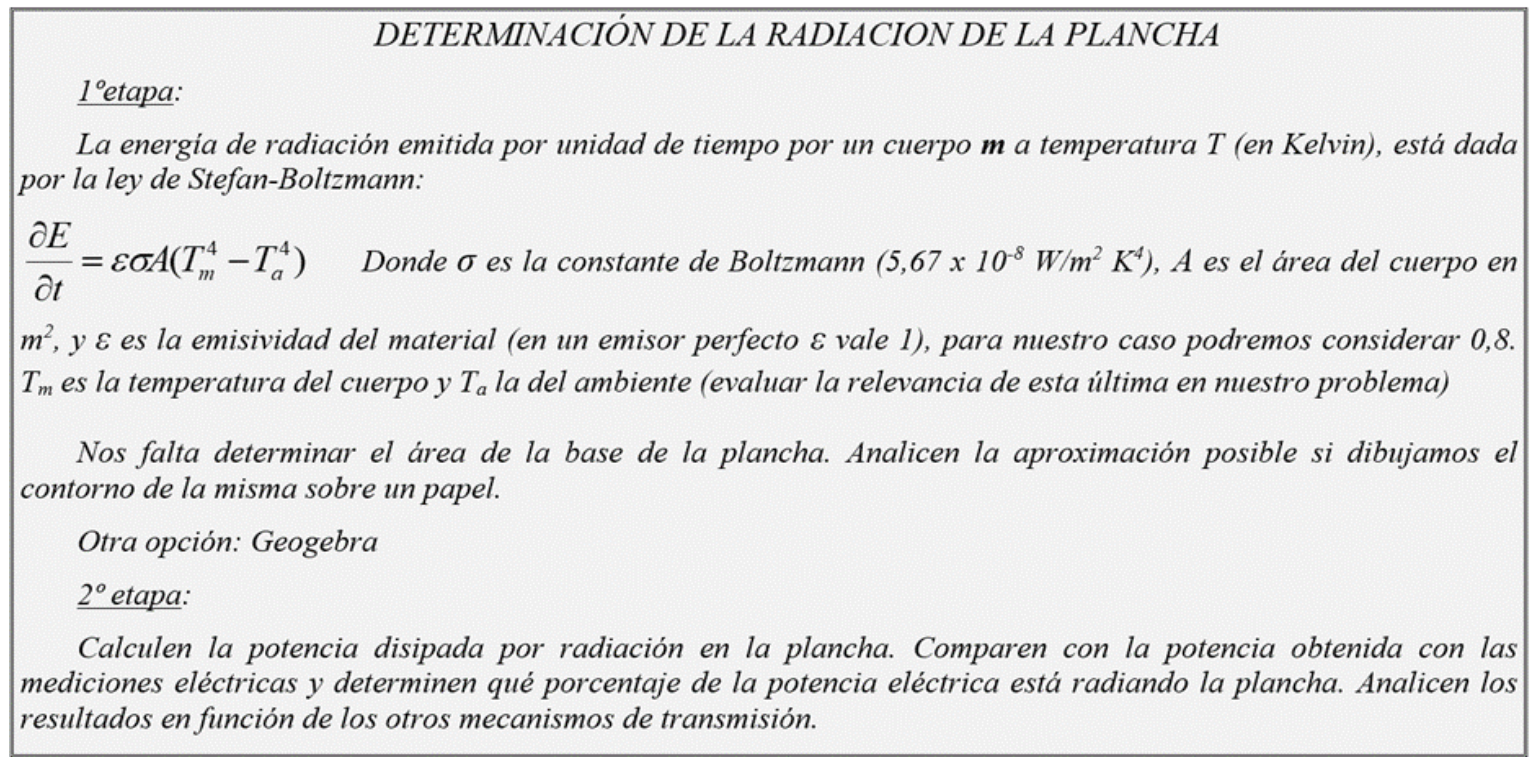

Figura 19. Apoyo teórico para la actividad B1

Pregunta hecha a los estudiantes:

¿Cómo podemos calcular, con buena aproximación, el área de una figura que no obedece al formato regular de una figura geométrica tradicional?

Para el cálculo de la radiación se requirió cuantificar el área de la superficie emisora. Si bien, en el proceso de modelización podría aproximarse a un triángulo, se propuso estimarla recurriendo a un antiguo método (aunque didáctico), que consiste en inscribir rectángulos dentro de la silueta de la base utilizando papel cuadriculado y sumar sus áreas correspondientes (ver figura 20, izquierda). Además de esto, se consideró una oportunidad de utilizar una función específica del software Geogebra. Con este programa pudo determinarse el área de la base a partir del área de su imagen (fotografiada en el laboratorio y mostrada en la figura 20, derecha) recurriendo a la función "polígono". Se convierten en $\mathrm{m}^{2}$ las unidades de área que brinda el programa planteando la proporción correspondiente a partir de una de sus dimensiones (con el programa también pueden medirse segmentos en unidades de longitud). 
La relación entre ambas áreas es proporcional al cuadrado de la relación entre una dimensión de la plancha y la correspondiente en la imagen.
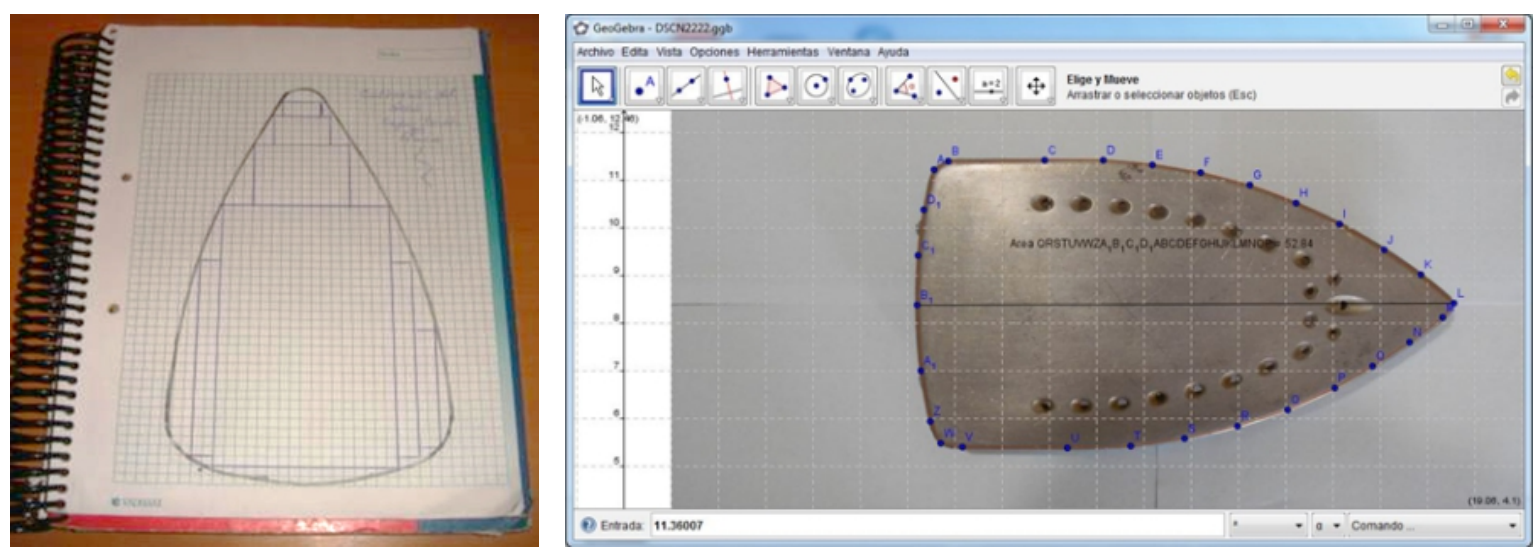

Figura 20. Izquierda: Determinación aproximada del área inscribiendo rectángulos dentro de la silueta en papel cuadriculado. La precisión del método dependerá de cuanto se desee aproximarse al área real. Derecha: Determinación del área con Geogebra. La precisión del resultado depende de la cantidad de lados con los que se decida construir el polígono que inscribe al borde de la imagen (los puntos en azul son los vértices).

Se consideró importante la discusión de la afirmación presentada al inicio del trabajo. No puede asumirse que el flujo entrante en un estado transitorio sea igual al flujo saliente debido a la variación continua de la energía interna de la plancha. El suministro de energía eléctrica no tiene un efecto inmediato en la temperatura. Además de la relación potencia/masa es la difusividad térmica la que gobierna este fenómeno. Este análisis permitió a los estudiantes tener una mayor comprensión del modelo que se estaba utilizando. Surgió del grupo una evaluación cualitativa haciendo uso del sensor, placa y software utilizado en la segunda actividad en común y tomando el tiempo de conexión eléctrica hasta el primer corte, partiendo de la temperatura ambiente. El resultado se muestra en la figura 21.

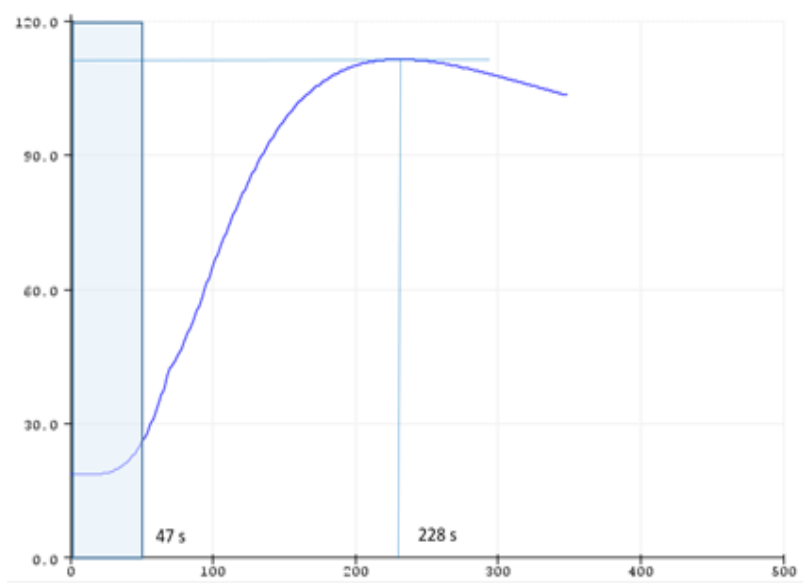

Figura 21. Gráfico obtenido por el grupo B. Se observa que el suministro eléctrico se produjo durante $47 \mathrm{~s}$ hasta el corte automático (parte sombreada) y la elevación de temperatura llega a su máximo a los $228 \mathrm{~s}$. La difusividad provoca que estos resultados no sean válidos para cualquier punto de la base de la plancha (para que el estado térmico evolucione naturalmente se desenchufó de la línea apenas producida la desconexión automática).

Para dar respuesta a la actividad B1 se reguló la temperatura "para prendas de algodón". En esta situación la misma fluctuó entre $176{ }^{\circ} \mathrm{C}$ y $188^{\circ} \mathrm{C}$ (medido con termopar). Debido a que el funcionamiento es intermitente se tuvo que determinar la potencia eléctrica media, a partir de la instantánea (midiendo tensión y corriente) y de cronometrar los intervalos de funcionamiento ( 7 segundos de calentamiento dentro de un período de 125 segundos). Para el cálculo de la radiación se consideró un modelo sencillo que tomó un valor de temperatura 
comprendido entre los dos anteriores; esto es equivalente a tomar la temperatura constante de un estado estacionario. De este modo se determinó que, para una plancha con una potencia superior a $1000 \mathrm{~W}$, la potencia radiada toma un valor sorprendentemente bajo, algo menos de $40 \mathrm{~W}$ para un área emisora de $0,013 \mathrm{~m}^{2}$, pero tal vez no lo sea tanto si se compara con la potencia media eléctrica media obtenida que fue de $107 \mathrm{~W}$ (apenas el $7 \%$ de la potencia instantánea medida). Con estos resultados la respuesta a la consigna inicial del grupo B es que la convección, restando a la potencia eléctrica media la de radiación y despreciando la conducción por el aire por ser un mal conductor del calor, alcanza algo más del $62 \%$. Naturalmente, un aspecto crucial de la enseñanza de la física que subyace en el balance de energía hecho con los estudiantes, es el de la conservación de la misma.

Resulta interesante, en términos de aprendizaje, la comparación de la potencia media cuando el planchado se lleva a cabo (actividad A1) y cuando no se hace (actividad B1). La primera prácticamente quintuplicó el valor de la segunda. Evidentemente la razón es que prevalece la conducción a través del contacto con la ropa.

\section{Actividad B2: La masa de las planchas}

La pregunta de la consigna se vinculó con aspectos más conceptuales presentes en la actividad. Se esperó que, de la discusión en el grupo B, surgieran relaciones entre las características constructivas de la plancha y ciertas variables termodinámicas involucradas.

Un menor peso implica menor masa, con lo cual -y esto es una ventaja funcional- disminuye el tiempo de calentamiento debido a que, para alcanzar una misma temperatura y para una misma potencia, es menor la energía interna necesaria en el electrodoméstico. Análogamente, el tiempo de enfriamiento también se verá disminuido, lo que resulta también ventajoso (para guardarla). Esto explicaría el criterio en los diseños actuales. Paralelamente se analizó por qué las planchas modernas tienen marcadamente más potencia eléctrica que las antiguas. Hubo acuerdo en que eso también disminuye los tiempos de calentamiento, pero sin comprometer el consumo. Efectivamente, mayor potencia no significa más energía consumida ya que sólo se consume la necesaria para compensar las "pérdidas" por calor, y la regulación de los tiempos de conexión eléctrica necesarios, los establece el interruptor automático. Esencialmente, la energía que se requiere para mantener un cuerpo a cierta temperatura en igualdad de condiciones de disipación es la misma. Por otra parte, las planchas actuales son construidas en aluminio en tanto que las antiguas eran de hierro; este dato, no conocido por los estudiantes, también se contempló para la discusión. El aluminio posee una difusividad térmica muy superior a la del hierro y eso otorga una clara ventaja para los tiempos de calentamiento. Todo este análisis cualitativo lo desarrolló el grupo B con el acompañamiento del docente. Tales consideraciones (en términos de la física) se relacionan con las actividades desarrolladas por el grupo A, lo cual facilitó vincular los trabajos de ambos en el cierre del trabajo.

\section{Actividad final de encuentro entre los dos grupos}

Al concluir el trabajo de los dos grupos sobre las situaciones A y B se propuso un intercambio de ideas donde se manifestaran los aprendizajes alcanzados sobre las problemáticas planteadas. Esto se desarrolló en el tramo final de la segunda clase de la secuencia de trabajo. Un integrante de cada grupo lideró la exposición de lo trabajado en forma oral, se compartieron aprendizajes, opiniones y se discutió también la viabilidad de transferencia de la actividad en el aula con alumnos de secundaria atendiendo a las posibles adaptaciones necesarias. Después se solicitó la elaboración de informes grupales con algunas pautas establecidas en cuanto al género científico y otras disposiciones en términos comunicativos como el ordenamiento de los apartados. Desde la materia se intenta promover la escritura en formatos de comunicación científica. 


\section{Consideraciones finales}

La secuencia de actividades se planificó del modo en que aquí se presenta y pudo desarrollarse según lo esperado. Requirió acompañamiento docente de modo bastante sostenido, aunque esto era algo previsto por lo particular de la propuesta. La actividad B1 fue la que en términos relativos requirió más asistencia, muy posiblemente por tener un mayor trabajo de modelización.

En términos más generales consideramos que fue beneficiosa la poca cantidad de estudiantes que participaron (8). Éstos valoraron muy positivamente el trabajo resaltando el eje vertebrador que fue el abordaje de temas de la física problematizándolos en un artefacto cotidiano. Resaltaron que no habían considerado previamente la posibilidad de tomar alguno de estos elementos para contextualizar la enseñanza. Según sus propios enunciados este enfoque permite "pensar sobre situaciones reales y resolverlas". También manifestaron ver potencialidad didáctica a la metodología como para ser implementada en el nivel medio, donde podrían tomar diferentes objetos como casos de estudio. El caso elegido, por ser un electrodoméstico que aparentemente no despierta demasiada curiosidad en el hogar, sumó algunas reflexiones en ese sentido. Consideramos que una de las fortalezas de la actividad es la modelización, tanto física como matemática y que permite ser resuelta con las TIC. Se asume que las actividades de aprendizaje en un formato de ABP como el propuesto, enriquecen la formación docente, favorecen el aprendizaje de los estudiantes y promueven otras formas de trabajo, algo más cercanas a las metodologías científicas. Esto puede resultar de gran valor para la futura práctica docente de los estudiantes de la carrera.

Una posible ventaja de la actividad es que, al tomar un artefacto que no es tradicional en la enseñanza, no requiere dispositivos específicos de laboratorio. Quizá pueda considerarse como tal el hardware para medir temperatura. Sin embargo, éste es de fácil adquisición y resulta económico. Se reconoce que fueron muy necesarios los multímetros; sobre éstos se advierte que para la medición de corriente resulta necesario que esto sea posible en alterna, ya que los de bajo costo no suelen tener esa función. La plancha a utilizar puede ser de cualquier modelo, aunque quizá el contar con una variedad de artefactos contribuiría a ampliar una caracterización construida desde los conceptos físicos. Pero debe tenerse en cuenta que no deben conectarse varias en simultáneo por el elevado consumo eléctrico que generan.

\section{Referencias}

Baker M. J. (2009) Intersubjective and Intrasubjective Rationalities in Pedagogical Debates: Realizing What One Thinks, pp. 145-158 en Schwarz, B., Dreyfus, T. y Hershkowitz, R. (eds.). Transformation of Knowledge through Classroom Interaction. Londres: Routledge.

Bennett J., Lubben F., Hogarth S. (2007) Bringing science to life: A synthesis of the research evidence on the effects of context-based and STS approaches to science teaching. Science Education 91 (3), 347-370.

Crujeiras B., Jiménez M. P. (2015) Desafíos planteados por las actividades abiertas de indagación en el laboratorio: articulación de conocimientos teóricos y prácticos en las prácticas científicas. Enseñanza de las Ciencias 33 (1), 63-84.

Cyrulies E. (2017) Actividades de capacitación docente en física utilizando un automóvil. Revista Brasileira de Ensino de Física. Vol 39 n 4.

Domènech Casal J. (2013) Secuencias de apertura experimental y escritura de artículos en el laboratorio: un itinerario de mejora de los trabajos prácticos en el laboratorio, Enseñanza de las Ciencias 31 (3), 249-262. 
Gairín Sallan J. (2011) Formación de profesores basada en competencias. Bordón. Revista de pedagogía 63 (1), 93-108.

Guisasola J., Ceberio M., Almudi J. M., Zubimendi J. L. (2011) La resolución de problemas basada en el desarrollo de investigaciones guiadas en cursos introductorios de física universitaria. Enseñanza de las Ciencias 29 (3), 439-452.

Hacking I. (1983). Representing and intervening. Cambridge: Cambridge University Press.

Martínez Torregrosa J., Gil D., Becerra C., Guisasola J. (2005) ¿Podemos mejorar la enseñanza de la resolución de problemas de «lápiz y papel» en las aulas de Física y Química? Educación química 16 (2), 230-245.

Perrenoud P. (2004) Diez nuevas competencias para enseñar (1 edic. en español). España: Graó, Biblioteca del Aula. Serie Didáctica/Diseño y desarrollo curricular.

Pifarré M., Sanuy J. (2001) La enseñanza de estrategias de resolución de problemas matemáticos en la ESO: Un ejemplo concreto. Enseñanza de las Ciencias 19 (2), 297-308.

Pozo J., Carretero M. (1987) Del pensamiento formal a las concepciones espontáneas: ¿Qué cambia en la enseñanza de las ciencias? Infancia y aprendizaje 38, 35-52.

Reiser B. J. (2004) Scaffolding Complex Learning: The Mechanisms of Structuring and Problematizing Student Work. The Journal of the Learning Sciences 13 (3), 273-304.

Rodríguez-Arteche I., Martínez-Aznar M.M. (2016) Introducing inquiry-based methodologies during initial secondary education teacher training using an open- ended problem about chemical change. Journal of Chemical Education 93 (9), 1528-1535.

Séré M. (2000) La enseñanza en el laboratorio. ¿Qué podemos aprender en términos de conocimiento práctico y de actitudes hacia la ciencia? Enseñanza de las ciencias 20 (3), 357-36.

Tejada Fernández J. (2009) Competencias docentes. Profesorado. Revista de Currrículum y formación del profesorado 13 (2), 222-234.

Tenreiro Vieira C., Marques Vieira R. (2006) Diseño y validación de actividades de laboratorio para promover el pensamiento crítico de los alumnos. Revista Eureka sobre Enseñanza y Divulgación de las Ciencias 3 (3), 452-466. 